\title{
Prevalence of Multiple White and Flat Elevated Lesions in Individuals Undergoing a Medical Checkup
}

\author{
Kyoichi Adachi ${ }^{1}$, Tomoko Mishiro ${ }^{1}$, Mayumi Okada ${ }^{1}$ and Yoshikazu Kinoshita ${ }^{2}$
}

\begin{abstract}
:
Objective The pathogenesis of multiple white and flat elevated lesions in the stomach has not been elucidated. We investigated the prevalence of such lesions and their characteristics in affected individuals.

Methods The subjects were 1,995 individuals (1,320 men, 675 women; mean age 54.2 \pm 9.5 years) who visited our medical center for a comprehensive annual medical checkup and in whom the status of Helicobacter pylori infection could be determined. The presence of multiple white and flat elevated lesions in the stomach and the degree of gastric mucosal atrophy were evaluated using endoscopic findings.

Results Multiple white and elevated lesions in the stomach were observed in 60 subjects (3.0\%), who were predominantly women and older in comparison to those without such lesions. The prevalence rates of these lesions in $H$. pylori-positive and $H$. pylori-negative and in post-eradicated subjects were $0.5 \%, 1.3 \%$, and $4.6 \%$, respectively. A multiple logistic regression analysis demonstrated that post-eradication status, female gender, older age, and a higher grade of gastric mucosal atrophy were significant risk factors for the occurrence of multiple white and elevated lesions.

Conclusion Multiple white and elevated lesions were frequently observed in subjects with successful $H$. $p y$ lori eradication.
\end{abstract}

Key words: multiple white and flat elevated lesions, stomach, Helicobacter pylori, eradication, proton pump inhibitor

(Intern Med 57: 1213-1218, 2018)

(DOI: 10.2169/internalmedicine.9808-17)

\section{Introduction}

The presence of multiple white and flat elevated lesions in the stomach was initially reported by Kawaguchi et al. in 2007 (1). Since then, such lesions have been repeatedly observed in the fundic gland area of individuals without Helicobacter pylori infection who had received anti-secretory drugs (2-4). The histological findings of these lesions have shown hyperplastic changes of the fundic gland foveolar epithelium, although the pathogenesis and significance of the condition remains unclear (1-4).

The national health insurance system of Japan began coverage in February 2013 for $H$. pylori eradication therapy to treat $H$. pylori-associated chronic gastritis, which is known to be closely associated with development of atrophic gastri- tis and gastric cancer (5), and the number of patients undergoing such eradication therapy has been rapidly increasing. Studies using endoscopic observation of the stomach have reported the presence of map-like redness or depressed patchy redness, along with an absence of diffuse redness, mucosal swelling, sticky mucous, and enlarged folds, in patients with successful $H$. pylori eradication $(4,6-8)$. We have also found multiple white and flat elevated lesions in the stomach of patients who have undergone successful eradication.

We therefore investigated the prevalence of multiple white and flat elevated lesions and their related characteristics in healthy individuals who came to our medical center for a comprehensive annual medical checkup.

${ }^{1}$ Health Center, Shimane Environment and Health Public Corporation, Japan and ${ }^{2}$ Second Department of Internal Medicine, Shimane University Faculty of Medicine, Japan

Received: July 11, 2017; Accepted: August 29, 2017; Advance Publication by J-STAGE: December 27, 2017

Correspondence to Dr. Kyoichi Adachi, adachi@kanhokou.or.jp 

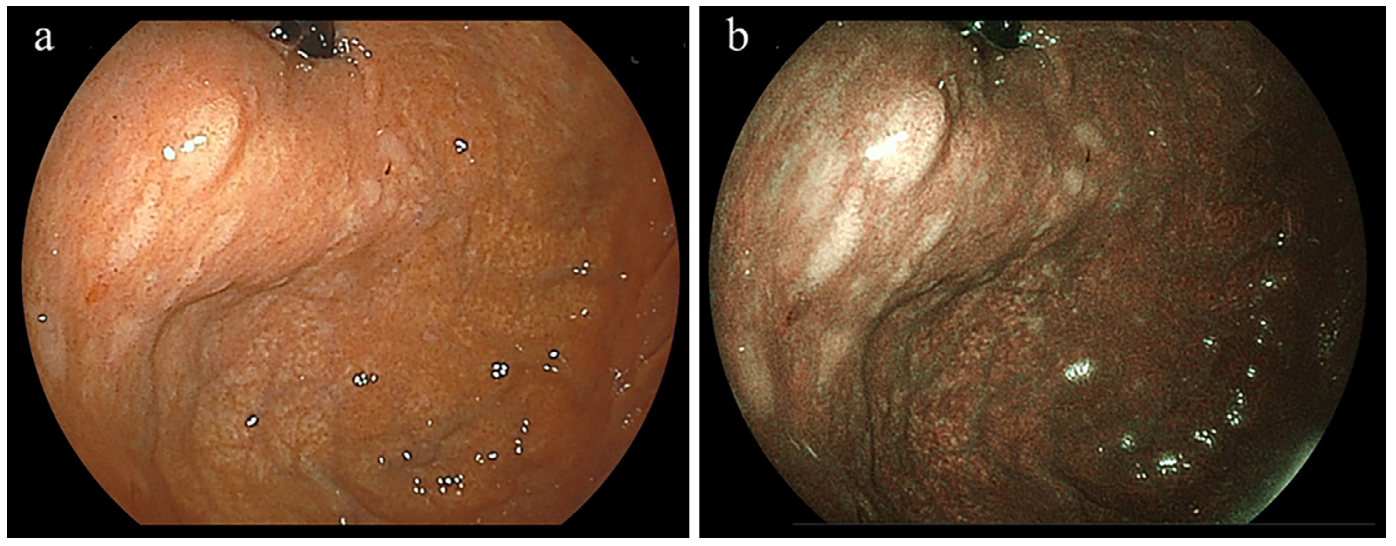

Figure 1. Representative case, 79-year-old woman who underwent eradication therapy for $H$. pylori 2 years prior to the examination. a: Endoscopy revealed multiple white and elevated lesions in the fornix of the stomach. b: The lesions were easily detected by endoscopic observations with blue laser imaging.
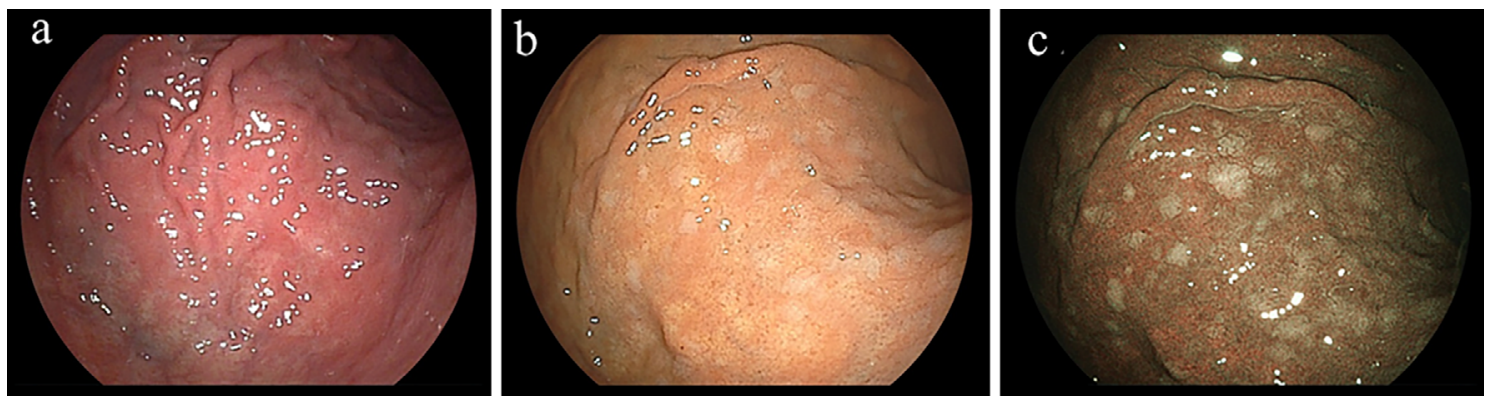

Figure 2. Representative case, 59-year-old woman. a: Endoscopy did not detect multiple white and elevated lesions in the upper body of the stomach prior to eradication of $\boldsymbol{H}$. pylori. b, c: Lesions were observed by endoscopy in the upper body of the stomach at seven months after successful $\mathrm{H}$. pylori eradication (blue laser imaging result shown in c).

\section{Materials and Methods}

The study subjects were individuals who visited the Health Center of Shimane Environment and Health Public Corporation for an annual medical checkup examination between April 2015 and March 2016. The majority were socially active and productive and considered to be socioeconomically middle class. Those with a history of gastric surgery were excluded. For this study, we selected cases in which the status of $H$. pylori infection was determined by medical records and/or serum antibody test findings to clarify the relationship between the prevalence of multiple white and flat elevated lesions and the status of $H$. pylori infection. A precise medical history concerning the status of $H$. pylori infection (negative, positive, post-eradication) was obtained in an interview conducted by a public health nurse. The use of medications of anti-secretory drugs, such as proton pump inhibitor and $\mathrm{H} 2$ receptor antagonist, within the preceding 12 months was also noted. Those without successful eradication were included in the group with $H$. pylori infection, even though they had undergone eradication therapy. When eradication therapy was confirmed as not successful, we recommended that the patient undergo an $H$. pylori stool antigen test at our institution. The presence or absence of $H$. pylori infection and successful eradication were also confirmed from endoscopic findings obtained on an upper gastrointestinal (GI) endoscopic examination, which was performed for all of the subjects (4, 6-9). The population was composed of 1,995 individuals (1,320 men, 675 women; mean age $54.2 \pm 9.5$ years) in whom the status of $H$. pylori infection was determined.

All upper endoscopic examinations were performed by licensed experienced endoscopists using an EG-L580NW or EG-530NP endoscope (Fujifilm, Tokyo, Japan). Multiple white and flat elevated lesions in the stomach are typically detected in the fornix and greater curvature of the upper body of the stomach. When the existence of such lesions could not be definitely determined, we performed imageenhanced endoscopy using a blue laser imaging or flexible spectral Imaging color enhancement device (Fig. 1-3). The degree of gastric mucosal atrophy was then evaluated using the classification of Kimura and Takemoto, in which gastric mucosal atrophy is divided into six categories $(\mathrm{C} 1, \mathrm{C} 2, \mathrm{C} 3$, O1, O2, O3) (10). For this study, we defined C1-C2 as mild, $\mathrm{C} 3-\mathrm{O} 1$ as moderate, and $\mathrm{O} 2-\mathrm{O} 3$ as severe gastric mucosal 

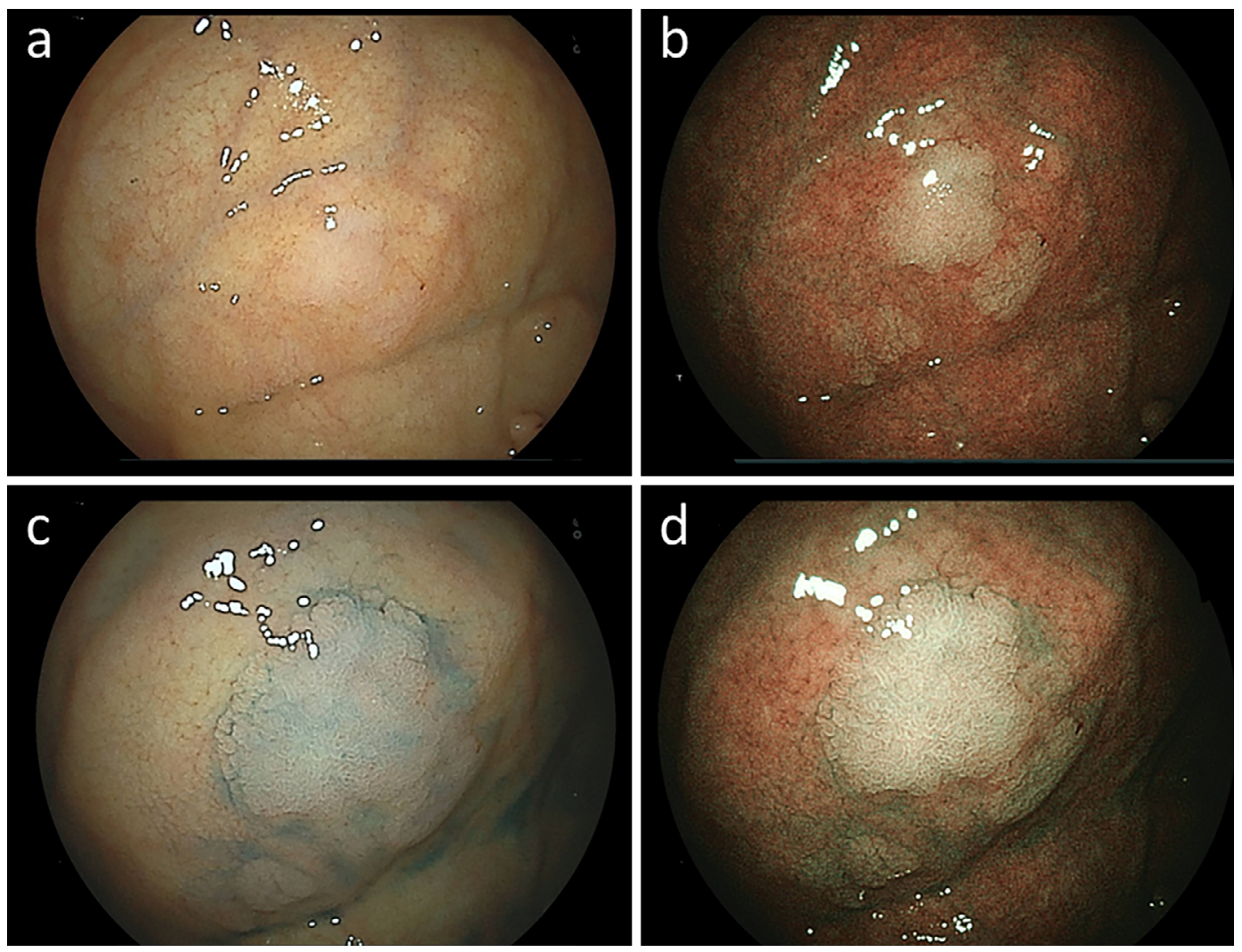

Figure 3. Representative case, 67-year-old woman without $H$. pylori infection and history of antisecretory drug use. a-d: Endoscopy revealed multiple white and elevated lesions in the fornix of the stomach (b: blue laser imaging, c: imaging after spraying indigo carmine dye, d: blue laser imaging after spraying indigo carmine dye).

atrophy. All of the endoscopic images from each subject were simultaneously reviewed by three licensed endoscopists, and the diagnosis of each endoscopic finding was made. If there were any inconsistencies in the reading of the endoscopic images between the three endoscopists, the final diagnosis was decided by one endoscopist (K.A.).

Statistical analyses were performed using a chi-squared test and Mann-Whitney U test. A multiple logistic regression analysis was also performed to calculate the odds ratios after adjusting for confounding factors. The Stat View software program, 5.0 (Abacus Concepts, Berkeley, USA), for Macintosh was used to perform the statistical analyses. $\mathrm{p}$ values $<0.05$ were considered to be statistically significant.

This study was performed in accordance with the Declaration of Helsinki and approved by the Ethics Committee of Shimane Institute of Health Science. Written informed consent indicating that clinical data without the release of individual information would be used for a clinical study was obtained from all subjects prior to performing the medical checkup examinations.

\section{Results}

Among the 1,995 study subjects, 1,103 underwent eradication for $H$. pylori, of whom 524 were originally $H$. pyloripositive, and 368 were originally $H$. pylori-negative. Multi- ple white and elevated lesions in the stomach were observed in $60(3.0 \%)$, in the area of the fornix and body of the stomach in all cases. The characteristics of subjects with and without such lesions are shown in Table 1. Those with the lesions were predominantly women and older than those without. The prevalence rates of multiple white and elevated lesions in $H$. pylori-positive and $H$. pylori-negative subjects were $0.5 \%$ and $1.3 \%$, respectively, while that in subjects after undergoing eradication for $H$. pylori was $4.6 \%$. Therefore, the lesions were more frequently observed in subjects after eradication for $H$. pylori than in those with an $H$. pylori-positive or $H$. pylori-negative status. Of all subjects with multiple white and elevated lesions, $85 \%$ had an $H$. pylori post-eradication status (Fig. 4). We were able to investigate the endoscopic images before $H$. pylori eradication in 42 of 51 cases with multiple white and elevated lesions and H. pylori post-eradication status. However, none showed multiple white and elevated lesions before $H$. pylori eradication. A higher grade of gastric mucosal atrophy and history of medication with anti-secretory drugs were characteristics of subjects with these lesions. Multiple white and elevated lesions were observed in 4 of 63 subjects with proton pump inhibitor use and 1 of 9 subjects with $\mathrm{H} 2$ receptor antagonist use. When the correlation between the prevalence of lesions and duration after eradication for $H$. pylori were analyzed in subjects who underwent eradication, the lesions were ob- 
Table 1. Characteristics of Subjects with and without Multiple White and Elevated Lesions.

\begin{tabular}{|c|c|c|c|}
\hline Multiple white and elevated lesions & Present & Absent & $\mathrm{p}$ value \\
\hline Male/female & $30 / 30$ & $1,290 / 645$ & 0.007 \\
\hline Age in years & $57.8 \pm 8.9$ & $54.1 \pm 9.5$ & 0.004 \\
\hline Habitual drinking $(+/-)$ & $24(40.0)$ & $931(48.1)$ & 0.215 \\
\hline Habitual smoking & $7(11.7)$ & $360(18.6)$ & 0.172 \\
\hline H. pylori infection & & & $<0.001$ \\
\hline Negative & $7(1.3)$ & $517(98.7)$ & \\
\hline Positive & $2^{\# \#(0.5)}$ & $366(99.5)$ & \\
\hline Eradicated & $51(4.6)^{*, * *}$ & $1,052(95.4)$ & \\
\hline Gastric mucosal atrophy & & & 0.004 \\
\hline Mild & $18(1.5)$ & $1,185(98.5)$ & \\
\hline Moderate and severe & $42(5.3)$ & $750(94.7)$ & \\
\hline History of anti-secretory drug use $\mathrm{e}^{\#}$ & $5(8.3)$ & $67(3.4)$ & 0.046 \\
\hline \multicolumn{4}{|c|}{$\begin{array}{l}\text { Data are expressed as the mean } \pm \mathrm{SD} \text {. Gastric mucosal atrophy was evaluated using the } \\
\text { classification of Kimura and Takemoto }(\mathrm{C} 1 \sim \mathrm{C} 2 \text { : mild, } \mathrm{C} 3 \sim \mathrm{O} 1 \text { : moderate, } \mathrm{O} 2 \sim \mathrm{O} 3 \text { : sever } \\
\text { gastric mucosal atrophy).\#: The numbers of subjects with proton pump inhibitor and } \mathrm{H} 2 \\
\text { receptor antagonist use were } 63 \text { and } 9 \text {, respectively. Numbers in parentheses indicate } \\
\text { percentage.\#\#: Female case with } \mathrm{C} 2 \text { gastric mucosal atrophy and male case with } \mathrm{O} 2 \text { gas } \\
\text { tric mucosal atrophy. Both cases did not have histories of eradication therapy for } H \text {. py } \\
\text { lori infection and anti-secretory drug use. * and } * * \text { indicate }<<0.05 \text { in comparison with } \\
\text { H. pylori-negative and -positive subjects, respectively. }\end{array}$} \\
\hline
\end{tabular}

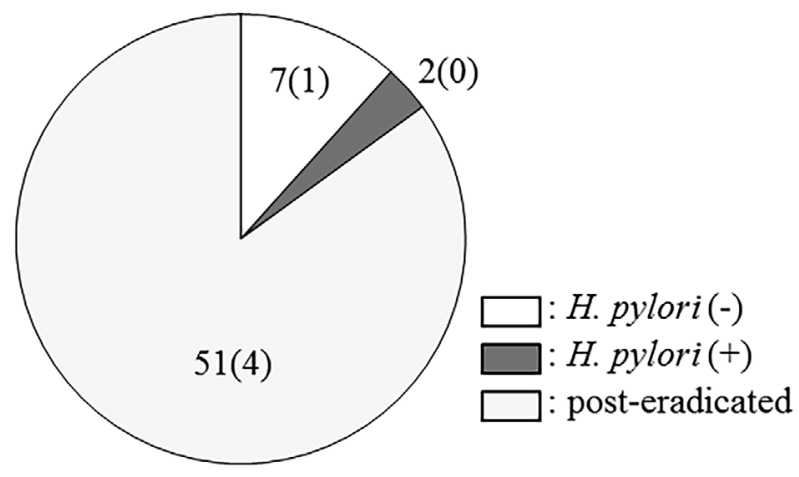

Figure 4. H. pylori infection status in subjects with multiple white and elevated lesions. Numbers in parentheses indicate subjects with a history of anti-secretory drugs administration. Of the subjects who had undergone eradication of $\mathrm{H}$. pylori, $85 \%$ had these lesions.

served at a variety of time periods after successful eradication (Table 2).

A multiple logistic regression analysis demonstrated that female gender, older age, and post-eradication status were significant factors for the occurrence of multiple white and elevated lesions. Negative status for $H$. pylori infection and a history of medication with anti-secretory drugs tended to be risk factors for the prevalence of the lesions (Table 3). A multiple logistic regression analysis of only subjects with successful eradication showed that a higher grade of gastric mucosal atrophy and female gender were significant risk factors for the occurrence of multiple white and elevated lesions (Table 4).
Table 2. Prevalence of Multiple White and Elevated Lesions, and Time of Occurrence after H. pylori eradication.

\begin{tabular}{lc}
\hline Period following eradication & Positive for lesions \\
\hline$<1$ year $(n=133)$ & $6(4.5)$ \\
$1 \sim 2$ years $(n=297)$ & $22(7.4)$ \\
$2 \sim 6$ years $(n=340)$ & $14(4.1)$ \\
$6 \sim 10$ years $(n=163)$ & $5(3.0)$ \\
$>10$ years $(n=170)$ & $4(2.4)$ \\
\hline
\end{tabular}

Numbers in parentheses indicate percentage.

\section{Discussion}

In this study, we investigated the occurrence of multiple white and elevated lesions in the stomach of subjects who visited our center for an annual medical checkup. Previously, such lesions in the stomach were reported to be mainly observed in the fundic gland area of subjects without H. pylori infection and who had received anti-secretory drugs (1-4). However, multiple white and elevated lesions were observed in $4.6 \%$ of the present subjects who had undergone eradication of $H$. pylori. To our knowledge, these are the first results to report the frequent occurrence of these lesions in the stomach following successful $H$. pylori eradication.

Previous studies have reported that the histology of multiple white and elevated lesions in the stomach indicated hyperplastic changes of the foveolar epithelium of the fundic gland (1-4), although the reason for their appearance in the stomach has not been clarified. One possible mechanism 
Table 3. Multiple Logistic Regression Analysis for Prevalence of Multiple White and Elevated Lesions.

\begin{tabular}{lccc}
\hline Factors & Odds ratio & $95 \%$ CI & p value \\
\hline Gender (female) & 2.256 & $1.240-4.105$ & 0.008 \\
Age (increment of 1 year) & 1.031 & $1.001-1.063$ & 0.044 \\
Habitual drinking & 0.886 & $0.492-1.597$ & 0.688 \\
Habitual smoking & 0.798 & $0.343-1.855$ & 0.599 \\
H. pylori infection (vs. positive) & & & \\
$\quad$ Negative & 2.459 & $0.506-11.958$ & 0.265 \\
$\quad$ Post-eradication & 8.477 & $2.044-35.162$ & 0.003 \\
History of anti-secretory drug use & 2.351 & $0.885-6.248$ & 0.087 \\
\hline
\end{tabular}

Table 4. Multiple Logistic Regression Analysis for Prevalence of Multiple White and Elevated Lesions in Cases Following Eradication of $\boldsymbol{H}$. Pylori.

\begin{tabular}{lccc}
\hline \multicolumn{1}{c}{ Factors } & Odds ratio & $95 \%$ CI & p value \\
\hline Gender (female) & 2.852 & $1.465-5.551$ & 0.002 \\
Age (increment of 1 year) & 1.018 & $0.983-1.055$ & 0.322 \\
Habitual drinking & 0.958 & $0.499-1.837$ & 0.897 \\
Habitual smoking & 0.860 & $0.342-2.160$ & 0.748 \\
Gastric mucosal atrophy\# & 2.928 & $1.522-5.634$ & 0.001 \\
History of anti-secretory drug use & 2.496 & $0.819-7.607$ & 0.108 \\
\hline
\end{tabular}

\#The odds ratio for moderate and severe gastric mucosal atrophy was calculated in comparison with mild gastric mucosal atrophy.

may be hypergastrinemia, since these lesions were reported to be frequently observed in patients who received proton pump inhibitor administration, which was also noted in the present subjects (3). However, hypergastrinemia has not been confirmed to cause multiple white and elevated lesions, nor has the presence of such lesions been demonstrated in patients with Zollinger-Ellison syndrome. In our study, those lesions were more frequently observed in subjects with successful eradication of $H$. pylori and a higher grade of gastric mucosal atrophy. Since increased gastric acid secretion in $H$. pylori-positive patients with a high grade of gastric mucosal atrophy has been reported following the successful eradication of $H$. pylori (11), the level of gastrin in serum is considered to be decreased after eradication therapy in those patients, suggesting that hypergastrinemia may not be a primary factor for the development of multiple white and elevated lesions. However, we were unable to clearly explain why multiple white and elevated lesions develop after eradication of $H$. pylori. The histology of this lesion shows hyperplastic changes of the foveolar epithelium; we therefore believe that several mechanisms involving stimulating the regeneration of gastric epithelium may be correlated with the occurrence of multiple white and elevated lesions. Further studies are needed to clarify the mechanism underlying the development of these lesions.

In the present cohort, 53 of 60 subjects with multiple white and elevated lesions had a continuous $H$. pylori infection or had undergone $H$. pylori eradication. However, 51 $(96.2 \%)$ were post-eradication cases, and only 2 (3.8\%) were found to be positive for an $H$. pylori infection. Therefore, these lesions are considered to appear after successful eradication of $H$. pylori. Interestingly, these lesions were observed in a variety of different time periods after eradication had been performed, suggesting that the period after successful eradication is not an important factor influencing their generation. Nevertheless, additional studies are needed to clarify the significance of the appearance of these lesions following eradication therapy for $\mathrm{H}$. pylori.

Older age and female gender were both risk factors for the prevalence of multiple white and elevated lesions. An older age may be related to a high degree of gastric mucosal atrophy, as that was also found to increase the risk of the lesions. However, why female gender was a significant risk factor was unclear, although several studies have noted a female predominance among subjects with such lesions (1-4). Further studies of the relationship between the levels of sex hormones in serum and the prevalence of multiple white and elevated lesions should be conducted.

Our study is limited by its cross-sectional nature. In addition, the one-year period of observation was short and did not allow us to investigate time-course endoscopic changes for the prevalence of multiple white and elevated lesions. A time-course endoscopic study should be performed to clarify the relationship between the eradication of $H$. pylori and the prevalence of these lesions. The majority of our study subjects were healthy individuals who visited a medical center for an annual medical checkup examination. Thus, the number of subjects with a history anti-secretory drug administra- 
tion was small, and the effects of that on the prevalence of multiple white and elevated lesions could not be determined. Many subjects in this study had $H$. pylori post-eradicated status. In our institute, we advised only those patients with positive endoscopic findings for $H$. pylori infection to undergo the serum antibody test, and we actively recommended that $H$. pylori-positive individuals receive eradication therapy. In this study, we excluded subjects whose $H$. pylori infection status was not determined by tests for $H$. pylori infection. Therefore, the number of $H$. pylori-negative cases was relatively small in this study. A further large-scale study is needed to determine the prevalence of multiple white and elevated lesions in cases without $H$. pylori infection.

In conclusion, the $H$. pylori post-eradication status, female gender, and older age were significant factors for the appearance of multiple white and elevated lesions. In addition, a higher grade of gastric mucosal atrophy was also a significant risk factor in subjects who had undergone successful eradication.

The authors state that they have no Conflict of Interest (COI).

\section{Acknowledgement}

We wish to thank Ms. Yoko Komori, Ms. Kiyoe Kawase, Ms. Yuki Funaki, Ms. Noriko Yamauchi and Mr. Takahiro Hatanaka in Shimane Environment and Health Public Corporation, and Ms. Rika Tohma and Ms. Keiko Masuzaki in Second Department of Internal Medicine, Shimane University Faculty of Medicine for their technical support.

\section{References}

1. Kawaguchi M, Arai E, Nozawa H, Kawamura H, Kitabora T.
White flat elevated lesions in the body of stomach. Gastroenterol Endosc 49 (Suppl 1): 958, 2007 (in Japanese).

2. Haruma K, Shiotani A, Kamada T, et al. Adverse effects induced by long-term use of proton pump inhibitor -development of gastric polyp. Clin Gastroenterology 56: 190-193, 2013 (in Japanese).

3. Kamada T, Haruma K, Takao T. Multiple white flat lesions (Haruma and Kawaguchi lesion). Endoscopia Digestiva 28: 1182-1183, 2016 (in Japanese).

4. Kyoto classification of gastritis. 1st ed. Haruma K, Kato M, Inoue K, Murakami K, Kamada T, Eds. Nihon Medical Center, Tokyo, 2014 (in Japanese).

5. Asaka M, Kato M, Sakamoto N. Roadmap to eliminate gastric cancer with Helicobacter pylori eradication and consecutive surveillance in Japan. J Gastroenterol 49: 1-8, 2014.

6. Watanabe $\mathrm{K}$, Nagata $\mathrm{N}$, Nakashima R, et al. Predictive findings for Helicobacter pylori-uninfected, -infected and -eradicated gastric mucosa: validation study. World J Gastroenterol 19: 43744379, 2013.

7. Kato M, Terao S, Adachi K, et al. Changes in endoscopic findings of gastritis after cure of $H$. pylori infection: multicenter prospective trial. Dig Endosc 25: 264-273, 2013.

8. Kamada T, Haruma K, Inoue K, Shiotani A. Helicobacter pylori infection and endoscopic gastritis -Kyoto classification of gastritis. Nihon Shokakibyo Gakkai Zasshi 112: 982-993, 2015 (in Japanese, Abstract in English).

9. Kato T, Yagi N, Kamada T, et al. Diagnosis of Helicobacter pylori infection in gastric mucosa by endoscopic features: a multicenter prospective study. Dig Endosc 25: 508-518, 2013.

10. Kimura K, Takemoto T. An endoscopic recognition of the atrophic border and its significance in chronic gastritis. Endoscopy 3: 8797, 1969

11. Iijima K, Ohara S, Sekine H, et al. Changes in gastric acid secretion assayed by endoscopic gastrin test before and after Helicobacter pylori eradication. Gut 46: 20-26, 2000.

The Internal Medicine is an Open Access article distributed under the Creative Commons Attribution-NonCommercial-NoDerivatives 4.0 International License. To view the details of this license, please visit (https://creativecommons.org/licenses/ by-nc-nd/4.0/).

\section{(C) 2018 The Japanese Society of Internal Medicine Intern Med 57: 1213-1218, 2018}

